Pacific Journal of Mathematics

THE SEIFERT AND VAN KAMPEN THEOREM VIA REGULAR ( 


\title{
THE SEIFERT AND VAN KAMPEN THEOREM VIA REGULAR COVERING SPACES
}

\author{
R. J. KNILL
}

The Seifert and Van Kampen theorem has lately been phrased as the solution to a universal mapping problem. There is given here an analogous theorem for regular covering spaces, regarded as principal bundles with discrete structure groups. The universal covering space of a union of two spaces is built up from the universal covering spaces of the two subspaces by an application of the associated bundle and clutching constructions. When all spaces are semi-locally simply connected, the Seifert and Van Kampen theorem is a consequence.

The technique of building a covering space piece by piece was effectively exploited by Neuwirth [10] to construct nonsimply connected covering spaces. We give an alternative approach to constructing a regular covering space of a base $B$ which is either the union of open sets $B_{1}$ and $B_{2}$ with connected intersection $B_{0}$, or which is an adjunction space $B_{1} \cup_{f} B_{2}$, where $f$ glues a closed subspace $B_{0}$ of $B_{1}$ to $B_{2}$. We assume that all spaces are connected, and that there is given a regular covering space $\xi_{i}$ of $B_{i}$ for $i=0,1$, and 2, together with morphisms of covering spaces $\xi_{0} \rightarrow \xi_{i}, i=1,2$. By regarding a regular covering space as a principal bundle with discrete structure group and applying the associated bundle and clutching constructions, we obtain a regular covering space $\xi$ of $B$ as the pushout of $\xi_{1} \leftarrow \xi_{0} \rightarrow \xi_{2}$. The structure group of $\xi$ is the pushout of the structure groups of $\xi_{0}, \xi_{1}$, and $\xi_{2}$. One may obtain in this fashion the universal covering space of $B$ from universal covering spaces of $B_{0}, B_{1}$, and $B_{2}$, or one may obtain the universal abelian covering space (i.e., that one having the maximal possible abelian structure group) from the universal abelian covering spaces of $B_{0}, B_{1}$, and $B_{2}$. The proof is by universal mapping arguments. In contrast to Neuwirth [10], the Seifert and Van Kampen theorem, under the hypotheses that all base spaces are locally connected and semi-locally simply connected, is a corollary. It is interesting that local homotopy conditions in a neighborhood of $B_{0}$, such as those assumed by Van Kampen and others ([15], [11], and [2]), turn out to be unnecessary, provided the space $B_{1}$ is paracompact. On the other hand, the Van Kampen formulae may apply in cases where $B_{0}$ is not connected or one of the $B_{i}$ does not have a universal covering space, neither case being included in our results.

The only difficult arguments involve the validity of the clutching construction [14], [1], and [7] for $B_{1} \cup_{f} B_{2}$. That is to say, one must 
establish the existence of a local product structure for any bundle formed by clutching. In the alternative case of open $B_{1}$ and $B_{2}$, with $B=B_{1} \cup B_{2}$, the clutching construction easily yields a locally trivial object. Thus the person interested in the simplest route to a form of Seifert and Van Kampen will consider only the case of open $B_{1}$ and $B_{2}$, with $B=B_{1} \cup B_{2}$.

The author wishes to acknowledge that the formulation in terms of universal properties was suggested by the referee.

1. Universality of the associated bundle. If $\xi$ is a principal bundle with structure group $G$, and $u: G \rightarrow K$ is a continuous group homomorphism, then $G$ acts on $K$ on the left via $u$, and the associated bundle construction yields a new bundle with structure group $K$. This associated bundle is the solution to a certain universal mapping problem in the category of all principal bundles.

All spaces are assumed to be regular. Recall [14] that a principal bundle $\xi$ consists of bundle space $E_{\xi}$, a base space $B_{\xi}$, a projection $p_{\xi}: E_{\xi} \rightarrow B_{\xi}$. There is also given a topological structure group $G_{\xi}$ which acts on $E_{\xi}$ freely from the right and such that $p_{\xi}$ is equivalent map of $E_{\xi}$ onto the space of orbits of $G_{\xi}$. It is assumed that $p_{\xi}$ is locally trivial. This means that there is an open covering $\left\{V_{i}\right\}_{i \in I}$ of $B_{\xi}$ by sets called coordinate neighborhoods, and for each $i \in I$ there exists a map (= continuous function) $s_{i}: V_{i} \rightarrow E_{\xi}$. This map is assumed to be a section of $\xi$ over $V_{i}$, that is, for any point $b$ of $V_{i}, p_{\xi}\left(s_{i}(b)\right)=$ $b$. Local triviality is the condition that the function $S_{i}$ defined below is a homeomorphism.

$$
S_{i}: V_{i} \times G_{\xi} \longrightarrow p_{\xi}^{-1}\left(V_{i}\right), \quad S_{i}(b, g)=s_{i}(b) \cdot g \cdot
$$

Here $s_{i}(b) \cdot g$ denotes the right translate of $s_{i}(b)$ by an element $g$ of $G$. The functions $S_{i}$ are called coordinate functions. The coordinate function determined by any section of $\xi$ is a homeomorphism.

LEMMA 1.1. If $\xi$ is a principal bundle and $f$ and $h$ are maps of a space $X$ into $E_{\xi}$ such that $p_{\xi}^{0} f=p_{\xi}^{0} h$, then there is a unique map $t: X \rightarrow G_{\xi}$ such that for any point $x$ of $X$, equation (1) holds

$$
f(x)=h(x) \cdot t(x) \text {. }
$$

Proof. For any point $x$ of $X$, equation (1) determines $t(x)$ uniquely, by the freeness of the action of $G_{\xi}$ on $E_{\xi}$. The map $t$ is continuous by local triviality of $p_{\xi}$.

DeFinition 1.2. Let $\xi$ and $\xi^{\prime}$ be principal bundles. A morphism 
$h: \xi \rightarrow \xi^{\prime}$ is a map of $E_{\xi}$ into $E_{\xi^{\prime}}$ such that there exists a function $h_{G}: G_{\xi} \rightarrow G_{\xi^{\prime}}$, which for any point $x$ in $E_{\xi}$, and element $g$ of $G_{\xi}$, satisfies equation (2)

$$
h(x \cdot g)=h(x) \cdot h_{G}(g) \cdot
$$

By the lemma, $h_{G}$ is unique and continuous. It is an easy consequence of (2) that $h_{G}$ is a homomorphism. Note that (2) also implies that $h$ maps a fibre $p_{\xi}^{-1}(b)$ into a fibre $p_{\xi}^{-1}\left(h_{B}(b)\right)$. Let $h_{B}: B \rightarrow B^{\prime}$ be the unique and continuous function such that for any point $x$ of $E_{\xi}$, equation (3) holds.

$$
p_{\xi^{\prime}}(h(x))=h_{B}\left(p_{\xi}(x)\right) .
$$

Let $\xi$ be a principal bundle, let $K$ be a topological group, and let $u: G_{\xi} \rightarrow K$ be a continuous homomorphism. Then $G_{\xi}$ acts on $K$ on the left via $u$, and the "weakly" associated bundle with fibre $K$ will be denoted by $\alpha_{u}$. See $[14, \S \S 8.7,9.1]$. The bundle space of $\alpha_{u}$ is usually denoted by $E \times{ }_{G} K$, where $E=E_{\xi}$. It is formed as the quotient space of $E \times K$ by the relation which identifies a point $(x, k)$ with $\left(x \cdot g, u\left(g^{-1}\right) k\right)$, for every element $g$ of $G_{\xi}$. The equivalence class of $(x, k)$ is denoted $\langle x, k\rangle$. The action of $K$ on $E \times{ }_{G} K$ is defined by the rule $\left\langle x, k_{1}\right\rangle \cdot k_{2}=\left\langle x, k_{1} k_{2}\right\rangle$. The base space of $\alpha_{u}$ is that of $\xi, B_{\alpha_{u}}=$ $B_{\xi}$. The projection is defined by the rule $p_{\alpha_{u}}\langle x, k\rangle=p_{\xi}(x)$. There is a natural map $u^{\sharp}: E \rightarrow E \times{ }_{G} K$ defined by the rule $u^{\sharp}(x)=\langle x, e\rangle$, where $e$ is the identity element of $K$. If $u$ is understood, then it will be convenient to write $\xi \times{ }_{G} K$ for $\alpha_{u}$.

THEOREM 1.3. Let $\xi$ be a principal bundle and let $u: G_{\xi} \rightarrow K$ be a continuous homomorphism of topological groups. Then $u^{\sharp}$ is a morphism of principal bundles such that $u_{G}^{*}=u$. If $h: \xi \rightarrow \xi^{\prime}$ is a morphism of principal bundles and $v: K \rightarrow G_{\xi^{\prime}}$ is a continuous homomorphism such that $h_{G}=v \circ u$, then there exists a unique morphism

$$
(h, v)^{\sharp}: \xi \times{ }_{G} K \rightarrow \xi^{\prime}
$$

such that (4) and (5) hold.

$$
\begin{aligned}
& h=(h, v)^{*} \circ u^{*} \\
& v=(h, v)_{G}^{*} .
\end{aligned}
$$

Proof. For $x$ in $E_{\xi}$ and $g$ in $G_{\xi}, u^{\sharp}(x \cdot g)=\langle x \cdot g, e\rangle=\langle x \cdot g, e\rangle=$ $\langle x, u(g)\rangle=\langle x, e\rangle \cdot u(g)=u^{\sharp}(x) \cdot u(g)$. This proves that $u^{\sharp}$ is a morphism and $u_{G}^{\sharp}=u$. The conditions (4) and (5) on $(h, v)^{\#}$ are equivalent by (2) to defining

$$
(h, v)^{\sharp}\langle x, k\rangle=h(x) \cdot v(k), \quad\langle x, k\rangle \in E \times{ }_{G} K .
$$


The uniqueness of $(h, v)^{\#}$ follows also from this rule, so the proof is complete.

Roughly that theorem says that $h: \xi \rightarrow \xi^{\prime}$ factors through $\alpha_{u}$ if and only if $h_{G}$ factors through $K$, and the former factorization is determined by the latter. In this sense the associated bundle is the solution to a universal mapping problem.

2. The clutching construction. The clutching construction ([14], p. 97) is extended. There are given spaces $B_{0}, B_{1}$, and $B_{2}$ such that $B_{0}$ is a subspace of $B_{1}$ with suitable properties relative to bundles. There is given a map $f: B_{0} \rightarrow B_{2}$ by means of which $B_{1}$ is attached to $B_{2}$ to form $B=B_{1} \cup_{f} B_{2}$ ([3], p. $\left.127 \mathrm{f}\right)$. There is given a common structure group $K$ for principal bundles $\xi_{0}, \xi_{1}$, and $\xi_{2}$ with respective base spaces $B_{0}, B_{1}$, and $B_{2}$ and there are given morphisms $j: \xi_{0} \rightarrow \xi_{1}$ and $h: \xi_{0} \rightarrow \xi_{2}$ such that $j_{B}: B_{0} \rightarrow B_{1}$ is the inclusion map and $h_{B}=f$, and such that $j_{G}$ and $h_{G}$ are the identity homomorphism of $K$. If $E_{i}$ is the bundle space of $\xi_{i}$ for $i=0,1$, and 2 , and let $E=E_{1} \cup_{h} E_{2}$. The projection $p$ of $E$ onto $B$ is defined by functoriality of the attaching construction, and likewise by functoriality $K$ acts on $E$ freely from the right. In order to conclude that $\xi=(E, p, B, K)$ is a principal bundle it suffices to prove that the projection is locally trivial. We write $\xi_{1} \cup_{h} \xi_{2}$ for $\xi$.

Definition 2.1. For any $\xi_{1}$ and $\xi_{2}$ as above, and for a closed subset $A$ of $B_{1}$, the set, $\mathscr{G}\left(A, \xi_{1}\right)$, of germs of sections of $\xi_{1}$ over $A$ is defined as follows. An element of $\mathscr{G}\left(A, \xi_{1}\right)$ is an equivalence class of sections of $\xi_{1}$ over neighborhoods of $A$, where two such sections, $s$ and $t$, are defined to be equivalent if for some open neighborhood $V$ of $A$ both $s$ and $t$ are defined throughout $V$ and their restrictions to $V$ are equal. For a closed subset $A$ of $B_{0}$, likewise there is defined $\mathscr{G}\left(A, \xi_{0}\right)$, and restriction of sections induces a function

$$
j^{*}: \mathscr{G}\left(A, \xi_{1}\right) \longrightarrow \mathscr{G}\left(A, \xi_{0}\right) \text {. }
$$

Let $\xi_{2}$ be a principal bundle, and let $f: B_{0} \rightarrow B_{\xi_{2}}$ be a map. Let $f^{*}(\xi)$ be the induced principal bundle $\left(f^{-1}(\xi)\right.$ in [14]) with base $B_{0}$, and let $\bar{f}: f^{*}\left(\xi_{2}\right) \rightarrow \xi_{2}$ be the canonical morphism. Recall that $\bar{f}_{B}=f, \bar{f}_{G}$ is the identity homomorphism, $\bar{f}_{G}: G_{f^{*}\left(\xi_{2}\right)}=G_{\xi_{2}}$, and it is easy to see that

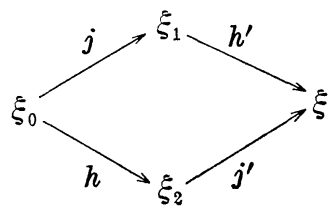

DIAGRAM I

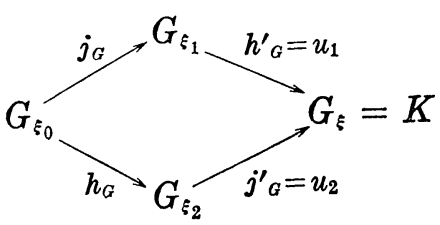

DIAGRAM II 


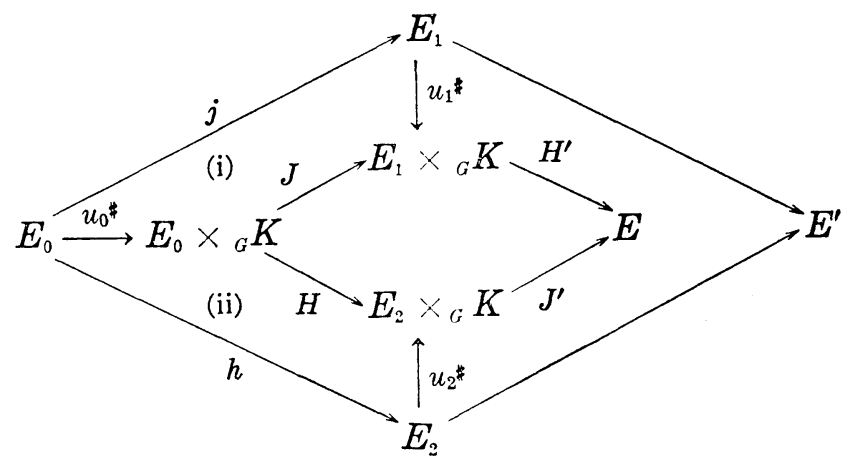

DIAGRAM III

these two conditions characterize $f^{*}\left(\xi_{2}\right)$. Recall further that for any section $s: V_{2} \rightarrow E_{\xi_{2}}$, where $V_{2}$ is a subset of $B_{\xi_{2}}$, there exists a unique section denoted $f^{*}(s): f^{-1}\left(V_{2}\right) \rightarrow E_{f^{*}\left(\xi_{2}\right)}$ such that $s \circ f=\bar{f}^{0} f^{*}(s)$.

TheOREM 2.2. Let $\xi_{0}, \xi_{1}, \xi_{2}, f, j, h$, and $\xi$ be as above. Let

$$
\begin{aligned}
& h^{\prime}: E_{1} \longrightarrow E_{1} \bigcup_{h} E_{2} \\
& j^{\prime}: E_{2} \longrightarrow E_{1} \bigcup_{h} E_{2}
\end{aligned}
$$

be the canonical maps. Under any of the conditions (2A), (2B), or (2C) stated below, $\xi$ is a principal bundle and $h^{\prime}$ and $j^{\prime}$ are morphisms of principal bundles which fill in the pushout diagram for $j$ and $h$ in the category of principal bundles (diagram I).

(2A) $B_{1}$ and $B_{2}$ are open subspaces of a common space $B=B_{1} \cup$ $B_{2}$, and $B_{0}=B_{1} \cap B_{2}$. The inclusion of $B_{0}$ into $B_{2}$ is $h_{B}=f$.

(2B) $B_{1}$ and $B_{2}$ are closed subspaces of a common space $B=B_{1} \cup$ $B_{2}$, and $B_{0}=B_{1} \cap B_{2}$. The inclusion of $B_{0}$ into $B_{2}$ is $h_{B}=f$. Further for each point $b$ of $B_{0}$ the restriction function of $\mathscr{G}\left(b, \xi_{1}\right)$ to $\mathscr{G}\left(b, \xi_{0}\right)$ is onto.

(2C) $B_{0}$ is a closed subspace of $B_{1}$, and $h_{B}=f$ is arbitrary. Further for every closed subset $A$ of $B_{0}$, the restriction function of $\mathscr{G}\left(A, \xi_{1}\right)$ to $\mathscr{G}\left(A, \xi_{0}\right)$ is onto. (Cf. 4.2 and 4.3).

Proof. For properties of the attaching construction see [3, pp. 127-129]. The canonical maps $h^{\prime}$ and $j^{\prime}$ are the restrictions to $E_{1}$ and $E_{2}$, resp., of the quotient projection of the free union, $E_{1}+E_{2}$, onto $E_{1} \cup_{h} E_{2}$. Assuming that $\xi$ is a principal bundle, then $h^{\prime}$ and $j^{\prime}$ fill in the pushout diagram ([9], p. 10) for $j$ and $h$, since they do so as maps in the category of topological spaces. It remains to show that $p$ is locally trivial. In case (2A), the coordinate neighborhoods for $p_{\xi_{1}}$ and $p_{\xi_{2}}$ are trivially coordinate neighborhoods for $p$. In case (2B), 
the coordinate neighborhoods for $p_{\xi_{1}}$ and $p_{\xi_{2}}$ in the complements of $B_{0}$ in $B_{1}$ and $B_{2}$, resp., are trivially coordinate neighborhoods for $p$. For an arbitrary point $b$ of $B_{1}$, let $V_{2}$ be an open neighborhood of $b$ relative to $B_{2}$, and let $s_{2}$ be a section of $\xi_{2}$ over $V_{2}$. Then $f^{*}\left(s_{2}\right)$ is the restriction of $s_{2}$ to a section, $s_{0}$, of $\xi_{0}$ over $V_{2} \cap B_{0}$. By hypothesis, the germ of $s_{0}$ over $b$ extends to a germ of a section $s_{1}$ of $\xi_{1}$ over a neighborhood $V_{1}$ of $b$ relative to $B_{1}$. By cutting down $V_{1}$ and $V_{2}$, if necessary, we may assume that $s_{1}, s_{0}$, and $s_{2}$ all are defined on $V_{1} \cap V_{2}$ and all agree there. Then $s_{1} \cup s_{2}$ is a section of $\xi$ over $V_{1} \cup V_{2}$, and the coordinate function defined by $s_{1} \cup s_{2}$ is a homeomorphism, by the functoriality of the attaching construction. In case (2C), the coordinate neighborhoods for $p_{\xi_{1}}$ in the complement of $B_{0}$ in $B_{1}$ are trivially coordinate neighborhoods for $p$. It remains to find coordinate neighborhoods of the points $b$ of $B_{2}$. So, let $s_{2}$ be a section of $\xi_{2}$ over an open neighborhood, $V_{2}$, of $b$. Let $A_{2}$ be a closed neighborhood of $b$ contained in $V_{2}$, and let $A=f^{-1}\left(A_{2}\right)$. Again $f^{*}\left(s_{2}\right)$ is a section, $s_{0}$, of $\xi_{0}$ in a neighborhood of $A$, and by hypothesis, the germ of $s_{0}$ over $A$ extends to a germ of a section, $s_{1}$, of $\xi_{1}$ over a neighborhood, $V_{1}$, of $A$ relative to $B_{1}$. By cutting down $V_{1}$ and $V_{2}$, if necessary, we may assume that $s_{1}$ and $s_{0}$ are both defined on $V_{1} \cap B_{0}$ and both agree there. Then $s_{1} \cup_{f} s_{2}$ is a section of $\xi$ (defined by functoriality of the attaching construction), the coordinate function defined by it being a homeomorphism by functoriality of the attaching construction. This completes the proof of theorem.

3. Universal covering spaces. The Seifert and Van Kampen theorem for regular covering spaces is the statement that pushouts exist in the category of regular covering spaces provided suitable conditions are satisfied by the base spaces. The construction does not generalize to locally compact principal fibre bundles since it would rely on the existence of pushouts in the category of locally compact structure groups.

A regular covering space of a connected base space $B$ is a principal bundle with base $B$ and a discrete structure group. The bundle space of a regular covering space is not assumed to be connected. The universal covering space of $B$ (if it exists) is the regular covering space such that for a fixed element $x_{0}$ of $E_{\xi}, \xi$ has the universality property: for any regular covering space $\xi^{\prime}$, for any map $f: B \rightarrow B_{\xi^{\prime}}$, and for any point $x^{\prime}$ of $E_{\xi^{\prime}}$ such that $p_{\xi^{\prime}}\left(x^{\prime}\right)=f\left(p_{\xi}\left(x_{0}\right)\right)$, there exists a unique morphism $f^{-}: \xi \rightarrow \xi^{\prime}$ such that $\left(f^{-}\right)_{B}=f$, and $f^{-}\left(x_{0}\right)=x^{\prime}$. It is not hard to conclude that the universal covering space of a given base space $B$ is unique up to isomorphism, that its bundle space is connected, that the universality property does not depend upon the choice of fixed element $x_{0}$, and that it suffices to verify the univer- 
sality property relative to covering spaces $\xi^{\prime}$ with the same base $B$ and for $f$ equal to the identity of $B$. It is also known that if a regular covering space of a base $B$ has a path connected simply connected bundle space, then it is the universal covering space of $B$, and its structure group is naturally isomorphic to the fundamental group of $B$. There exists an example ([13], p. 84 and [6]) of a connected, locally path connected metric space $B$ such that $B$ has a universal covering space which is not simply connected.

We now consider circumstances similar to those of paragraph 2. There are given spaces $B_{0}, B_{1}$, and $B_{2}$ such that $B_{0}$ is a subspace of $B_{1}$. There is given a map, $f$, of $B_{0}$ into $B_{2}$, by means of which $B_{1}$ is attached to $B_{2}$ to form $B=B_{1} \cup_{f} B_{2}$. There are given regular covering spaces $\xi_{0}, \xi_{1}$, and $\xi_{2}$ with respective base spaces $B_{0}, B_{1}$, and $B_{2}$. No assumption is made that the structure groups are isomorphic. There are given morphisms, $j$ and $h$, of $\xi_{0}$ to $\xi_{1}$, and of $\xi_{0}$ to $\xi_{2}$, respectively, such that $j_{B}$ is the inclusion map of $B_{0}$ into $B_{1}$, and such that $h_{B}=f$. Let $E_{i}$ be the bundle space of $\xi_{i}$, for $i=0,1$, and 2 . There exists a discrete group, $K$, and there exist morphisms, $u_{1}$ and $u_{2}$, of $G_{\xi_{1}}$ and $G_{\xi_{2}}$, respectively, into $K$ filling in the pushout diagram for $j_{G}$ and $h_{G}$ (diagram II). Let $u_{0}=u_{1} \circ j_{G}=u_{2} \circ h_{G}$. Using these homomorphisms define the associated bundles $\xi_{i} \times_{G} K=\alpha_{u_{i}}$ for $i=0,1$, and 2. Then $j$ and $h$ induce morphisms

$$
\begin{aligned}
J: E_{0} \times_{G} K \longrightarrow E_{1} \times_{G} K, & J(\langle x, k\rangle)=\langle j(x), k\rangle \\
H: E_{0} \times_{G} K \longrightarrow E_{2} \times_{G} K, & H(\langle x, k\rangle)=\langle h(x), k\rangle .
\end{aligned}
$$

Evidently $J_{G}=H_{G}=$ the identity homomorphism of $K$. Then define

$$
\begin{aligned}
E & =\left(E_{1} \times{ }_{G} K\right) \bigcup_{H}\left(E_{2} \times{ }_{G} K\right) \\
\xi & =\left(\xi_{1} \times{ }_{G} K\right) \bigcup_{H}\left(\xi_{2} \times{ }_{G} K\right) .
\end{aligned}
$$

There are natural maps $H^{\prime}$ and $J^{\prime}$ induced by the clutching construction filling in a pushout diagram of spaces (the inside cell of diagram III). Let $h^{\prime}=H^{\prime} \circ u_{1}^{\#}$ and $j=J^{\prime} \circ u_{2}^{\sharp}$. Then diagram I is a pushout diagram, provided that $\xi$ is a principal bundle. The theorem will be that this will be so in cases (3A), (3B), and (3C).

(3A) $\quad B_{1}$ and $B_{2}$ are open subspaces of a common space $B=B_{1} \cup B_{2}$, and $B_{0}=B_{1} \cap B_{2}$. The inclusion of $B_{0}$ into $B_{2}$ is $h_{B}=f$.

(3B) $B_{1}$ and $B_{2}$ are closed subspaces of a common space $B=$ $B_{1} \cup B_{2}$, and $B_{0}=B_{1} \cap B_{2}$. The inclusion of $B_{0}$ into $B_{2}$ is $h_{B}=f$.

(3C) $B_{0}$ is a closed subspace of $B_{1}, B_{1}$ is paracompact, and $h_{B}=$ $f$ is arbitrary.

Lemma 3.1. Case (3B) implies case (2B), and case (3C) implies 
case (2C), for the principal bundles $\xi_{i} \times{ }_{G} K$ over $B_{i}, i=0,1,2$.

Proof. In case (3B) note that since $K$ is discrete, then for a point $b$ of $B_{0}$ any two sections of $\xi_{0} \times{ }_{G} K$ which are defined over neighborhoods of $b$ must have the same germ over $b$. It follows that the restriction function of $\mathscr{G}\left(b, \xi_{1} \times{ }_{G} K\right)$ to $\mathscr{G}\left(b, \xi_{0} \times{ }_{G} K\right)$ is an isomorphism, and case (2B) holds. In case (3C), we regard all regular covering spaces to be sheaves of sets. Let $A$ be any closed subset of $B_{0}$. By a standard theorem of sheaf theory ([4], p. 150), any section over $A$ extends to a section over a neighborhood of $A$, and since two sections must agree over an open set, then it follows that the restriction function of $\mathscr{G}\left(A, \xi_{1} \times{ }_{G} K\right)$ to $\mathscr{G}\left(A, \xi_{0} \times{ }_{G} K\right)$ is an isomorphism and case $(2 \mathrm{C})$ holds.

TheOREM 3.2. Let $\xi_{0}, \xi_{1}, \xi_{2}, f, j, h$, and $\xi$ be as above. Under any of the conditions (3A), (3B), or (3C), diagram I is a pushout diagram in the category of regular covering spaces, and the induced diagram II of homomorphisms of structure groups is a pushout diagram in the category of groups.

Proof. Cells (i) and (ii) of diagram III are commutative as an application of (1.3). Let $h^{\prime}=H^{\prime} \circ u_{1}^{\#}$, and $j=J^{\prime} \circ u_{2}^{\#}$. Since $H_{G}^{\prime}$ and $J_{G}^{\prime}$ both are the identity homomorphism of $K$, then

$$
h_{G}^{\prime}=\left(u_{1}^{\sharp}\right)_{G}=u_{1},
$$

and

$$
j_{G}^{\prime}=u_{2} \text {. }
$$

We show that $h^{\prime}$ and $j^{\prime}$ fill in the pushout diagram for $j$ and $h$ in the category of regular covering spaces. Suppose that for $i=0,1$, and 2 , there are given morphisms $l_{i}: \xi_{i} \rightarrow \xi^{\prime}$ such that $l_{0}=j \circ l_{1}=h \circ l_{2}$. Since then

$$
\left(l_{0}\right)_{G}=\left(l_{1}\right)_{G} \circ j_{G}=\left(l_{2}\right)_{G} \circ h_{G}
$$

then there exists a unique homomorphism $v: K \rightarrow G_{\xi^{\prime}}$ such that

$$
v \circ h_{G}^{\prime}=l_{1},
$$

and

$$
v \circ j_{G}^{\prime}=l_{2} .
$$

For each $i=0,1$ or $2, l_{i}$ induces a unique morphism

$$
\left(l_{i}, v\right)^{\sharp}: \xi_{i} \times{ }_{G} K \longrightarrow \xi^{\prime}
$$


such that

$$
\left(l_{i}, v\right)_{G}^{\sharp}=v \text {, and }\left(l_{i}, v\right) \circ u_{i}^{\sharp}=l_{i} \text {. }
$$

It follows that

$$
\left(l_{1}, v\right)^{\sharp} \circ J=\left(l_{0}, v\right)^{\sharp}=\left(l_{2}, v\right)^{\sharp} \circ H .
$$

Since $H^{\prime}$ and $J^{\prime}$ fill in the pushout diagram for $J$ and $H$ then $\left(l_{1}, v\right)^{*}$ and $\left(l_{2}, v\right)^{\#}$ induce a unique morphism $l: \xi \rightarrow \xi^{\prime}$ such that

$$
\left(l_{1}, v\right)^{\sharp}=l \circ H^{\prime} \text {, and }\left(l_{2}, v\right)^{\sharp}=l_{\circ} J^{\prime} \text {. }
$$

By its construction, $l$ satisfies

$$
l \circ h^{\prime}=l_{1}, \text { and } l \circ j^{\prime}=l_{2}
$$

and $l$ is the unique morphism which does so. Since $l_{1}$ and $l_{2}$ were arbitrary such that $l_{1} \circ j=l_{2} \circ h$, this completes the proof that $h^{\prime}$ and $j^{\prime}$ fill in the pushout diagram for $j$ and $h$.

The Corollary 3.3 is the analogue for universal covering spaces of the Seifert and Van Kampen theorem.

COROLLARY 3.3. Under the conditions of 3.2, if $\xi_{i}$ is the universal covering space of $B_{i}$ for $i=0,1$, and 2 , then $\xi$ is the universal covering space of $B$, and the diagram II of induced homomorphisms of structure groups is a pushout diagram in the category of groups.

Proof. There exist morphisms $j: \xi_{0} \rightarrow \xi_{1}$, and $h: \xi_{0} \rightarrow \xi_{2}$ such that $j_{B}=$ the inclusion of $B_{0}$ into $B_{1}$, and $h_{B}=f$. Then there exist pushout diagrams in the category of covering spaces and in the category of groups. (See illustration I.) Let $\xi^{\prime}$ be any regular covering space of $B$, and suppose there are given points $x$ of $E_{\xi}$, such that $p_{\xi}(x)=$ $p_{\xi^{\prime}}\left(x^{\prime}\right)$. We must find a morphism $l: \xi \rightarrow \xi^{\prime}$ such that $l(x)=x^{\prime}$, and show that such a morphism is unique, in order to complete the proof. We may assume that $x$ is so chosen that there is a point $x_{0}$ in $E_{\xi_{0}}$ such that $h^{\prime}\left(j\left(x_{0}\right)\right)=x$. For $i=0,1$, and 2 , let $l_{i}: \xi_{i} \rightarrow \xi^{\prime}$ be the unique morphism such that $\left(l_{i}\right)_{B}$ is the natural map of $B_{i}$ into $B$ defined by the attaching construction, and such that $l_{0}\left(x_{0}\right)=x^{\prime}, l_{1}\left(j\left(x_{0}\right)\right)=x^{\prime}$, and $l_{2}\left(h\left(x_{0}\right)\right)=x^{\prime}$. Since they agree at one point, $x_{0}$, the maps $l_{0}, l_{1} \circ j$, and $l_{2} \circ h$ are all equal. Let $l$ be the unique morphism such that $l \circ h^{\prime}=l_{1}$, and $l \circ j^{\prime}=l_{2}$. This completes the proof.

Comment 3.4. Theorem 3.2 here stated and proven for all principal bundles with structure groups in the category of (discrete) groups, could be stated and be valid with no change in proof for any 
full subcategory of the category of principal bundles provided the corresponding category of structure groups had pushouts. The notion of universal principal bundle in that category would make sense provided the structure groups were discrete. For example, by taking the corresponding category of structure groups to be the category of abelian groups, one arrives at the notion of universal abelian regular covering space, and Theorems 3.2 and 3.3 in terms of abelian regular covering spaces would remain valid.

4. Extension properties of germs. The extension properties of germs are developed for principal bundles in general, there being interest in the validity of the clutching construction. There is a tradeoff in hypotheses to be made, it being necessary to strengthen hypotheses on the base spaces in order to admit less stringent conditions on the structure group.

Throughout this section $\xi_{1}$ is a principal bundle with base $B_{1}$ and structure group $K$, and $B_{0}$ is a closed subspace of $B_{1}$. Let $\xi_{0}=\xi_{1} \mid B_{0}=$ $j^{*}\left(\xi_{1}\right)$ be the restriction of $\xi_{1}$ to $B_{0}$, where $j$ is the inclusion of $B_{0}$ into $B_{1}$. Recall that for a closed subset $A$ of $B_{1}$, one says of $A$ that it has the neighborhood extension property in $B_{1}$ relative to $K$ provided that for any map $f$ of $A$ into $K$, there exists an extension of $f$ to a map of a neighborhood of $A$ into $K$.

THEOREM 4.1. If every closed subset of $B_{1}$ has the neighborhood extension property in $B_{1}$ relative to $K$, and if $B_{1}$ is paracompact, then case (2C) holds.

Proof. The proof is a straightforward modification of standard arguments which conclude a global property from the corresponding local property; see [14], p. 55, or [5], Theorem 2.7. To begin, let $V_{0}$ be a closed relative neighborhood of $A$ in $B_{0}$, and let $s_{0}$ be any section of $\xi_{0}$ over $V_{0}$. It will be shown that there exists an extension of $s_{0}$ to a section $s_{1}$ of $\xi_{1}$ over a closed neighborhood, $V_{1}$, of $V_{0}$ in $B_{1}$. That would suffice to prove the theorem. Let $\left\{U_{i}\right\}_{i \in I}$ be a locally finite open covering of $B_{1}$ by coordinate neighborhoods, and for each index $i$, let $s_{i}$ be a section of $\xi_{1}$ over $U_{i}$. Let $\left\{V_{i}\right\}_{i \in I}$ be a closed covering of $B_{1}$ such that for each index $i, V_{i}$ is contained in $U_{i}$. For any subset $J$ of $I$, let

$$
V_{J}=V_{0} \cup\left(\bigcup\left\{V_{i}: i \in J \text {, and } V_{0} \cap V_{i} \neq \varnothing\right\}\right) .
$$

Let $\mathscr{S}$ be the set of all pairs $(J, s)$, where $J$ is a subset of $I$, and $s$ is an extension of $s_{0}$ to a section of $\xi_{1}$ over a closed set, $N_{s}$, which is a relative neighborhood of $V_{0}$ in $V_{J}$. $\mathscr{S}$ is partially ordered by 
the relation defined as $(J, s) \leqq\left(J^{\prime}, s^{\prime}\right)$ if $J \subset J^{\prime}$ and $s^{\prime}$ extends $s$. By the Hausdorff maximal principal, there exists a maximal chain, $\mathscr{C}$, contained in $\mathscr{S}$. If we let

$$
J^{\prime}=\bigcup\{J:(J, s) \in \mathscr{C}\},
$$

and

$$
s^{\prime}=\bigcup\{s:(J, s) \in \mathscr{C}\},
$$

then $\left(J^{\prime}, s^{\prime}\right)$ is the maximum of $\mathscr{C}$. If $J^{\prime}=I$, then $N_{s^{\prime}}$ would be a neighborhood of $V_{0}$ in $B_{1}$, and the theorem would be proven. Suppose that there were an index $i$ not contained in $J^{\prime}$. Then $V_{i} \cap V_{0} \neq \emptyset$, for otherwise $\left(J^{\prime} \cup\{i\}, s^{\prime}\right)$ would be an element of $\mathscr{S}$ greater than $\left(J^{\prime}, s^{\prime}\right)$. Let

$$
t: N_{s^{\prime}} \cap V_{i} \longrightarrow K
$$

be the map defined by the equation

$$
s^{\prime}(x)=s_{i}(x) \cdot t(x), \quad x \in N_{s^{\prime}} \cap V_{i} .
$$

By the neighborhood extension property hypothesis, there is an extension, $t^{\prime}$, of $t$ to a map of a closed neighborhood, $M$, of $N_{s^{\prime}} \cap V_{i}$ into $K$. Then extend $s^{\prime}$ to a section $s^{\prime \prime}$ over $N_{s^{\prime}} \cup\left(M \cap V_{i}\right)$ by letting, for any point $x$ of $M \cap V_{i}$,

$$
s^{\prime \prime}(x)=s_{i}(x) \cdot t^{\prime}(x) .
$$

Then $\left(J^{\prime} \cup\{i\}, s^{\prime \prime}\right)$ is an element of $\mathscr{S}$ greater than $\left(J^{\prime}, s^{\prime}\right)$, contrary to the maximality of $\mathscr{C}$ and of $\left(J^{\prime}, s^{\prime \prime}\right)$. It follows that $J^{\prime}=I$. As previously observed, this proves the theorem.

COROLlaRY 4.2. If $B_{1}$ is a paracompact space, and $K$ is a Lie group, then case (2C) holds.

Proof. Since $K$ is topologically complete and an ANR, then [8] every closed subset of $B_{1}$ has the neighborhood extension property in $B_{1}$ relative to $K$, and so 4.1 implies 4.2 .

If the structure group, $K$, is not a Lie group, all is not lost.

THeOREM 4.3. If $\xi_{1}$ has the homotopy lifting property and $B_{0}$ is a neighborhood deformation retract of $B_{1}$, and in particular if the inclusion of $B_{0}$ into $B_{1}$ is a cofibration, then case (2C) holds.

Proof. This straightforward application of the homotopy lifting property is left to the reader. 


\section{REFERENCES}

1. M. Atiyah, K-Theory Lectures, Fall 1964, Harvard University, Cambridge.

2. R. Brown, Groupoids and Van Kampen's theorem, Proc. London Math. Soc., (3) 17 (1967), 385-401.

3. J. Dugundji, Topology, Boston, 1966.

4. R. Godement, Topologie algebrique et theorie des faisceaux, Hermann, Paris, 1958.

5. Albrecht Dold, Partitions of unity in the theory of fibrations, Ann. of Math., 78 (1963), 223-255.

6. H. B. Griffiths, The fundamental group of two spaces with a common point, Quarterly J. of Math., Oxford (2nd) Series, 5 (1954), 175-190.

7. Dale Husemoller, Fibre Bundles, McGraw-Hill, New York, 1966.

8. Ernest Michael, Some extension theorems for constructing functions, Pacific J. Math., 3 (1953), 789-806.

9. B. Mitchell, Theory of Categories, Academic Press, New York, 1965.

10. L. P. Neuwirth, Knot Groups, Annals of Mathematics Studies Number 56, Princeton, 1965.

11. P. Olum, Nonabelian cohomology and Van Kampen's theorem, Ann. of Math., (2) 68 (1958), 658-668.

12. H. Siefert and W. Threllfall, Lehrbuck der Topologie, Berlin, 1934.

13. E. Spanier, Algebraic Topology, New York, 1966.

14. N. Steenrod, The Topology of Fibre Bundles, Princeton University Press, Princeton, 1957.

15. E. R. Van Kampen, On the connection between the fundamental groups of some related spaces, Amer. J. Math., 55 (1933), 261-267.

Received June 23, 1972 and in revised form May 15, 1973. Research supported in part by NSF Grant \# GP 29707.

TUlane University 


\section{PACIFIC JOURNAL OF MATHEMATICS}

\section{EDITORS}

RICHARD ARENS (Managing Editor) University of California

Los Angeles, California 90024

R. A. Beaumon'T

University of Washington Seattle, Washington 98105
J. Dugundj1*

Department of Mathematics University of Southern California Los Angeles, California 90007

D. Gilbarg and J. Milgram Stanford University Stanford, California 94305

\section{ASSOCIATE EDITORS}

E. F. BECKENBACH

B. H. NeUMANN

F. WOLF

K. YOSHIDA

\section{SUPPORTING INSTITUTIONS}

UNIVERSITY OF BRITISH COLUMBIA CALIFORNIA INSTITUTE OF TECHNOLOGY UNIVERSITY OF CALIFORNIA MONTANA STATE UNIVERSITY UNIVERSITY OF NEVADA NEW MEXICO STATE UNIVERSITY OREGON STATE UNIVERSITY UNIVERSITY OF OREGON OSAKA UNIVERSITY

\section{UNIVERSITY OF SOUTHERN CALIFORNIA STANFORD UNIVERSITY UNIVERSITY OF TOKYO UNIVERSITY OF UTAH WASHINGTON STATE UNIVERSITY UNIVERSITY OF WASHINGTON AMERICAN MATHEMATICAL SOCIETY NAVAL WEAPONS CENTER}

* C. R. DePrima California Institute of Technology, Pasadena, CA 91109, will replace J. Dugundji until August 1974. 


\section{Pacific Journal of Mathematics}

\section{Vol. 49, No. $1 \quad$ May, 1973}

A. Bigard, Free lattice-ordered modules ...........................

Richard Bolstein and Warren R. Wogen, Subnormal operators in strictly cyclic

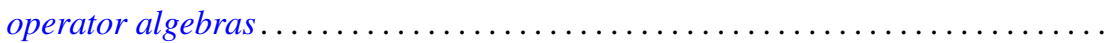

Herbert Busemann and Donald E. Glassco, II, Irreducible sums of simple

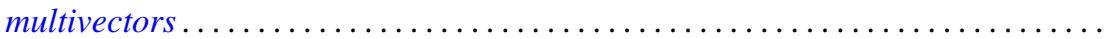

W. Wistar (William) Comfort and Victor Harold Saks, Countably compact groups

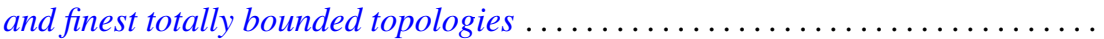

Mary Rodriguez Embry, Maximal invariant subspaces of strictly cyclic operator

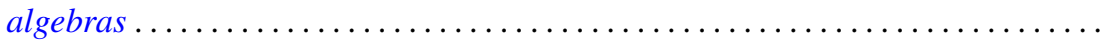

Ralph S. Freese and James Bryant Nation, Congruence lattices of semilattices......

Ervin Fried and George Grätzer, A nonassociative extension of the class of

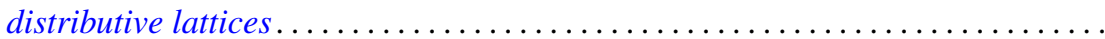

John R. Giles and Donald Otto Koehler, On numerical ranges of elements of locally

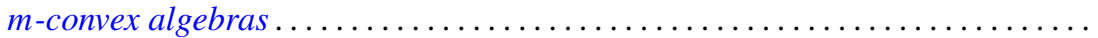

David A. Hill, On dominant and codominant dimension of $\mathrm{QF}-3$ rings ........ John Sollion Hsia and Robert Paul Johnson, Round and Pfister forms over $R(t) \ldots$ I. Martin (Irving) Isaacs, Equally partitioned groups . . . . . . . . . . . . . .

Athanassios G. Kartsatos and Edward Barry Saff, Hyperpolynomial approximation of solutions of nonlinear integro-differential equations.

Shin'ichi Kinoshita, On elementary ideals of $\theta$-curves in the 3-sphere and 2-links in the 4-sphere

Ronald Brian Kirk, Convergence of Baire measures

R. J. Knill, The Seifert and Van Kampen theorem via regular covering spaces ..

Amos A. Kovacs, Homomorphisms of matrix rings into matrix rings ..

Young K. Kwon, HD-minimal but no $H D$-minimal ..........

Makoto Maejima, On the renewal function when some of the mean renewal lifetimes are infinite

Juan José Martínez, Cohomological dimension of discrete modules over profinite groups.

W. K. Nicholson, Semiperfect rings with abelian group of units

Louis Jackson Ratliff, Jr., Three theorems on imbedded prime divisors of principal ideals.

Billy E. Rhoades and Albert Wilansky, Some commutants in $B(c)$ which are almost matrices

John Philip Riley Jr., Cross-sections of decompositions . . .

Keith Duncan Stroyan, A characterization of the Mackey uniformity $m\left(L^{\infty}, L^{1}\right)$ for

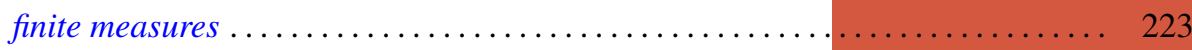

Edward G. Thurber, The Scholz-Brauer problem on addition chains . . . . . . . . . 229

Joze Vrabec, Submanifolds of acyclic 3-manifolds ............

Philip William Walker, Adjoint boundary value problems for compactified singular

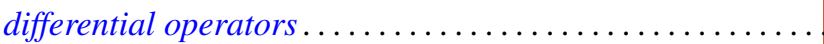

